\title{
SCIDiC
}

International Journal of Dentistry and Oral Science (IJDOS)

ISSN: 2377-8075

\section{The Effects Of Breakfast On Behavior Of Pediatric Patients In Dental Operatory}

Research Article

Deepa Gurunathan ${ }^{1 *}$ Lakshmi Lakshmanan ${ }^{2}$

${ }^{1}$ Professor Department of Pedodontics Saveetha Dental College and Hospitals Saveetha Institute of Medical and Technical Sciences Saveetha University, Chennai, India.

${ }^{2}$ Postgraduate student Department of Pedodontics Saveetha Dental College and Hospitals Saveetha Institute of Medical and Technical Sciences Saveetha University Chennai 77, India.

\section{Abstract}

Background: The greatest challenge faced by a pediatric dentist while treating a child in the dental operatory is the uncooperative behaviour. The aim of the study was to evaluate the effect of breakfast on behavior of pediatric patients in dental operatory.

Material and Methods: A cross-sectional study was conducted among 80 children aged between 4-10 years undergoing non invasive treatment. The children were divided in to two groups: did not skip breakfast (control group) and skipped breakfast (study group). Children's behavior was assessed using Frankl's behavior rating scale during the course of treatment (pretreatment, mid-treatment, and post-treatment). Descriptive statistics and independent $t$ tests were performed for analysis. Results: A statistically significant difference was noted in the behavior score between the two groups of children during the course of treatment $(\mathrm{P}<0.05)$. The overall mean score of children in group I was $3.26 \pm 0.2$ and in group II was $2.1 \pm 0.5$. The reasons for skipping breakfast were insufficient time (52.5\%), not being hungry (30\%), or believing in the myth to have an empty stomach prior to dental appointment $(17.5 \%)$.

Conclusion: A possible relation exists between breakfast consumption and behavior changes in young children.

Keywords: Behavior; Breakfast; Dental Operatory; Frankl's Behavior Rating Scale; Pediatric Dentist.

\section{Introduction}

Breakfast is the first meal of the day consisting of food or beverage from at least one food group which breaks the fast after the longest period of sleep and is consumed with in 2 to 3 hours of waking [1]. Breakfast is widely conceded as the most important meal of the day as it brings about metabolic changes involved in regulating blood glucose, insulin levels, lipid metabolism, appetite and energy balance [2].

Children have an increased brain glucose metabolism compared with adults. Positron Emission Tomography researchers indicate that cerebral metabolic rate of glucose utilization is relatively twice as high in children aged 4-10 years when compared with adults [3]. As the brain of a child is relatively larger and more active than the adult's, children may be particularly more responsive to provision of glucose which is considered as the major fuel of the brain [4]. Additionally, the longer overnight fasting period, due to higher sleep demands during childhood and adolescence, can deplete glycogen stores overnight [5]. In order to maintain this higher metabolic rate, a continuous supply of energy derived from glucose is required. Therefore, breakfast consumption may be paramount in providing adequate energy for day [6].

Sandercock et al., stated that children who do not devour breakfast are more likely to be less physically active and have a lower cardio-respiratory fitness level [7]. Moreover, there is evidence that breakfast consumption positively influences learning in chil-

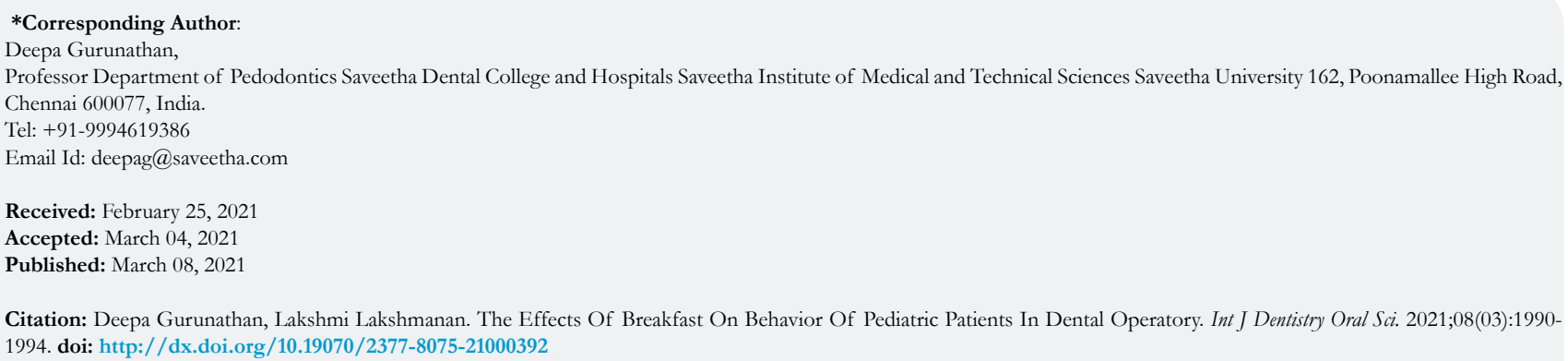

Copyright: Deepa Gurunathan ${ }^{\circ} 2021$. This is an open-access article distributed under the terms of the Creative Commons Attribution License, which permits unrestricted use, distribution and reproduction in any medium, provided the original author and source are credited. 
dren in terms of behavior, cognitive, and school performance [8]. The benefits of eating a breakfast meal have been reported extensively, with recent findings showing an immediate effect on cognitive performance, feeling of well-being, added to benefits related to nutrient intakes and diet quality $[8,9]$.

Although the evidence is quite mixed, studies demonstrate that eating breakfast has a positive effect on children in terms of memory, mood and attention [10, 11]. Adolphus et al., reported that the ignorance of morning meals will end up with the symptoms such as extreme hunger, irritation, anger, aggression and fatigue, due to exhaustion of glucose levels in the morning [12]. Consuming breakfast can have a positive effect on cortisol - the primary "stress hormone". The levels of cortisol are reported to be highest early in the morning, which necessitates the consumption of breakfast to bring down the hormone levels. If cortisol levels are too high, the children most likely feel anxious [13].

Pediatric dentists often have to face a battle before treating their patient, due to disruptive behavior [14]. Lack of cooperation in children not only affects the completion and quality of necessary dental treatment, but also aggravates the degree of stress in the dentist. Out of various reasons for externalizing behavior in children, skipping breakfast may be one. Though, this factor is not under the control of a practitioner, the knowledge about its influence on children's behavior can be of considerable aspect in clinical practice.

Various studies have evaluated the effects of breakfast on cognitive and academic performance in school children. To the best of our knowledge, none of the studies have assessed the influence of breakfast in behavior of patients in dental operatory. Our department is passionate about child care, and we have published numerous high quality articles in this domain over the past three years [15-26]. With this inspiration we planned to pursue research on the effects of breakfast on behavior of pediatric patients in dental operatory.

\section{Material and Methods}

The cross-sectional study was conducted in the Department of Pediatric and Preventive Dentistry from October 2019 to February 2020. Ethical approval for the study was obtained from the Institutional Scientific Review Board prior to the start of the study (IHEC/SDC-PEDO1803/19/036). The participation in the study was voluntary and informed consent was obtained from the parents or the care-takers of the children.

A pilot study was conducted among 20 children and the sample size for this study was determined by using G-Power analysis with a power of $80 \%$ and confidence interval $95 \%$ that arrived at a total sample of 80 .

The inclusion criteria were healthy children between the ages of 4-10 years who require non invasive treatment for primary or permanent teeth. Children with a history of any systemic disorder, on constant medication, undergoing invasive dental treatment, whose consent was not obtained from parents/guardians, or whose behavior modification has already been done, were excluded from the study.
Parents of these children were questioned regarding the consumption of breakfast: time, portion size and if skipped, the reason for skipping breakfast- were recorded. The children who were reported to have consumed any energy containing food or beverage from at least one food group with in 2-3 hours of waking were included as the control group (Group-I; Children who did not skip breakfast) and those who completely skipped the morning meal were included as the study group (Group-II; Children who skipped breakfast). The study was conducted early in the morning. All children were exposed to tell-show-do behavior management techniques.

In the dental operatory, the child's behavior was rated according to Frankl's behavior rating scale into definitely negative, negative, positive and definitely positive (Score 1-4) (Figure 1). The behavior of the children were recorded: (i) Before start of treatment (Pre-treatment) at the consulting room, (ii) When treatment was rendered (Mid-treatment) and (iii) Post-treatment before relieving the patient from the department. The behavior of children was assessed by a trained pediatric dentist other than the operator throughout the study.

\section{Statistical Analysis}

The extracted data were tabulated in a spreadsheet (Excel 2017: Microsoft Office) and analyzed using SPSS software 17.0 version (SPSS Inc., Chicago, IL, USA). Descriptive statistics and Independent $\mathrm{t}$ tests were done for analysing the data and a $\mathrm{P}$ value of $<0.05$ was said to be statistically significant.

\section{Results}

Of 80 children (Group I- 40; Group II- 40) participated in the study, 55\% (44 children) were males and 45\% (36 children) were females. The mean age of children in group I was $6.47 \pm 1.53$ years and in group II was $6.14 \pm 1.28$ years. The distribution of participants is shown in Table 1.

In terms of behavior assessment, a statistically significant difference was noted between the two groups of children during the course of treatment $(\mathrm{P}<0.05)$ (Table 2$)$. The overall mean score of children in group I was $3.26 \pm 0.2$ and in group II was $2.1 \pm$ 0.5 . The behavior of children participated in the study during the course of treatment is depicted in Figure 2.

Based on gender comparison, there was no statistically significant difference in both the groups during all phases of treatment $(\mathrm{P}>0.05)$ (Table 3).

The reasons stated for skipping breakfast were either insufficient time $(52.5 \%)$, not being hungry $(30 \%)$, or believing in the myth to have an empty stomach prior to dental appointment $(17.5 \%)$.

\section{Discussion}

In the pediatric dental service provision point of view, the longterm accomplishment of any treatment provided by a pediatric dentist profoundly relies upon the cooperation level of a child during the dental visit. Therefore, as much as it is essential for dentists to realize how to manage the children's behaviour in the dental operatory, it is imperative to know the factors that impact 
Table 1. Summary of demographic variables describing sample size, age (mean \pm standard deviation) and number of male and female participants in each group.

\begin{tabular}{|c|c|c|c|}
\hline Treatment Groups & Sample Size & Age (Years) & Gender (Female/Male) \\
\hline $\begin{array}{c}\text { Did not skip breakfast } \\
\text { (Control group) }\end{array}$ & 40 & $6.47 \pm 1.53$ & $20 / 20$ \\
\hline $\begin{array}{c}\text { Skipped breakfast } \\
\text { (Study group) }\end{array}$ & 40 & $6.14 \pm 1.28$ & $16 / 24$ \\
\hline
\end{tabular}

Table 2. Behavior rating scores (Mean \pm Standard deviation) during the course of treatment in the treatment groups.

\begin{tabular}{|c|c|c|c|}
\hline & $\begin{array}{c}\text { Did not skip breakfast (Control } \\
\text { group) }\end{array}$ & $\begin{array}{c}\text { Skipped breakfast } \\
\text { (Study group) }\end{array}$ & P value \\
\hline Pre-treatment & $3.5 \pm 0.6$ & $2.7 \pm 0.9$ & $<0.001^{*}$ \\
\hline Mid-treatment & $3.1 \pm 0.7$ & $1.7 \pm 0.7$ & \\
\hline Post-treatment & $3.2 \pm 0.6$ & $1.8 \pm 0.8$ & \\
\hline Overall Mean Score & $3.2 \pm 0.6$ & $2.1 \pm 0.5$ & \\
\hline
\end{tabular}

Table 3. Behavior rating scores (Mean \pm Standard deviation) and statistical comparison based on gender in the treatment groups.

\begin{tabular}{|c|c|c|c|c|c|c|}
\hline \multirow{2}{*}{ Gender } & \multicolumn{3}{|c|}{ Control Group } & \multicolumn{3}{c|}{ Study Group } \\
\cline { 2 - 7 } & $\begin{array}{c}\text { Pre- treat- } \\
\text { ment }\end{array}$ & $\begin{array}{c}\text { Mid- treat- } \\
\text { ment }\end{array}$ & $\begin{array}{c}\text { Post- treat- } \\
\text { ment }\end{array}$ & $\begin{array}{c}\text { Pre- treat- } \\
\text { ment }\end{array}$ & $\begin{array}{c}\text { Mid- treat- } \\
\text { ment }\end{array}$ & $\begin{array}{c}\text { Post- treat- } \\
\text { ment }\end{array}$ \\
\hline Female & $3.5 \pm 0.6$ & $3.1 \pm 0.7$ & $3.1 \pm 0.8$ & $2.5 \pm 1.1$ & $1.3 \pm 0.5$ & $1.5 \pm 0.7$ \\
\hline Male & $3.4 \pm 0.6$ & $3.1 \pm 0.8$ & $3.3 \pm 0.4$ & $2.8 \pm 0.7$ & $2.0 \pm 0.8$ & $1.9 \pm 0.9$ \\
\hline P value & 0.70 & 0.70 & 0.36 & 0.17 & 0.08 & 0.07 \\
\hline
\end{tabular}

$\mathbf{P}>0.05$; Not significant

Figure 1. Frankl behavior rating scale used in the study for assessing the behavior of study participants.

\begin{tabular}{|l|l|l|}
\hline \multicolumn{2}{|c|}{ FRANKL BEHAVIOR RATING SCALE } \\
\hline $\mathbf{1}$ & $\begin{array}{c}\text { ATIITUDE } \\
\text { DEFINITION }\end{array}$ \\
\hline $\mathbf{2}$ & Definitely negative & $\begin{array}{l}\text { Refusal of treatment, crying forcefully, fearful or } \\
\text { any other overt evidence of extreme negativism. } \\
\text { Reluctant to accept treatment, uncooperative, } \\
\text { some evidence of negative attitude but not } \\
\text { pronounced, i.e./ sullen, withdrawn. }\end{array}$ \\
\hline $\mathbf{3}$ & Negative & $\begin{array}{l}\text { Acceptance of treatment; at times cautious, } \\
\text { willingness to comply with the dentist, at times } \\
\text { with reservation but patient follows the dentist's } \\
\text { directions cooperatively. } \\
\text { Good rapport with the dentist, interested in the } \\
\text { dental procedures, laughing, and enjoying the } \\
\text { situation. }\end{array}$ \\
\hline
\end{tabular}

Figure 2. The image represents the behavior of children participated in the study during the course of treatment, where $\mathrm{Y}$-axis denotes the behavior rating score and $\mathrm{X}$-axis denotes the course of treatment. Blue color denotes Group 1 (Control group) and Red color denotes Group II (Study group). Drastic behavior change towards negative behavior can be noted in the study group when compared to the control group and the differences are statistically significant in all the phases of treatment (Independent $\mathrm{t}$ test; $\mathrm{P}=<0.001$, Statistically significant).

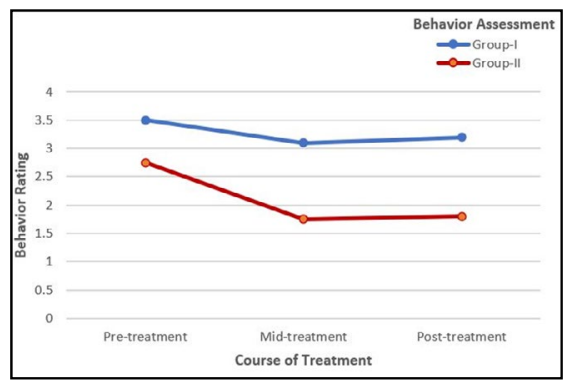

the behaviours [27]. Additionally, understanding the reason for children's behaviour assists the dentist to plan appointments and render effective and efficient dental treatment [28].
The uncooperative behavior in children could be attributed to a variety of reasons, among which skipping breakfast could be one. 
Kleinman et al, stated that children who encounter hunger are seven times more likely to immerse in physical altercations [29]. Additionally, several studies reported that hunger can lead to attention problems, behavior and emotional problems $[4,8,10]$. Hence, it is crucial to understand their role in the child's behavior. Till date, there is no published study in the literature that has assessed the relationship of breakfast with the child's behavior in the dental operatory. With this understanding, the present study was taken up to evaluate the effect of breakfast on the behavior of children in the dental operatory.

The current study utilized Frankl behavior rating scale for behavior assessment, which is one of the proven reliable tools developed for behavior rating of children in dental sittings [30].

Mishra et al., stated that the type of treatment rendered affects the behavior significantly as the children who under went non invasive procedures such as restorations, oral prophylaxis showed positive behavior and those who underwent invasive procedures like extraction and root canal treatment exhibited negative behavior [28]. The current study included children who required noninvasive dental treatment only to rule out the pain, needle phobia and other related factors that increases the children's apprehension which on its own promotes negativity and may contribute to negative behavior.

On an average, the children in the control group showed positive behaviour during the different phases of treatment. Where as in the study group, the proportion of children who displayed positive behaviour during the pre-treatment phase, decreased during the course of treatment. While those who showed negative behavior continued the same through out the course of treatment. It was also observed that the children in the study group exhibited more negative behaviour towards the end of the treatment. These findings are in agreement with those of Adolphus et al., that the ignorance of breakfast results in irritation, anger and aggression, thereby modifying the behaviour of children [12]. Moreover, Jamali et al., stated that the extended dental treatment length is associated with higher levels of behavioral problems in children [31]. The present study also divided children according to gender to identify if there is any significant difference of behavior between male and female and found no significant differences in both groups $(\mathrm{P}>0.05)$.

Various researchers have reported that a more positive behaviour can be expected from children as age increases, since older children are more likely to be emotionally mature to understand things and tend to cope with the circumstances [32, 33]. Conversely, in the study group, children despite age, exhibited negative and definitely negative behavior during the course of treatment. Skipping breakfast can make children feel tired, restless, or irritable. Breakfast is the way to refuel the body for the day ahead after going without food for 8 to 10 hours during sleep. The children's mood, energy, and the ability to pay attention can drop by midmorning if they don't consume at least a small morning meal [5, 10]. The attention span of children is of paramount importance in successfully completing the dental treatment; deficiency of which can lead to the use of advanced behavior guidance techniques such as sedation [34].

The explanations stated for skipping breakfast were insufficient time $(52.5 \%)$, not being hungry $(30 \%)$, or believing in the myth to have an empty stomach prior to dental appointment (17.5\%). These are in accordance with the study conducted by Fugas et al., which stated the lack of time, not being hungry in the morning and feeling unwell at the time of having breakfast as explanations for skipping breakfast before going to school [35]. These findings underline the need for stressing the importance of morning meals to parents and children.

The reason for uncooperative behavior of the children in the study group cannot be limited to only skipping breakfast. The behavior of the child on dental visits can be influenced by variables such as age, parental behavior, parental anxiety, past medical and dental history, type of dental settings, treatment duration, behavior management and procedural techniques followed by the dentist [36].

The major limitations of this study were the limited sample size, short study duration and also related to the fact that this was not a random sample of patients scored by independent observers, rather the patients reporting for their dental operatory visit who were treated by a single dentist and also scored by another single pediatric dentist. Intra-examiner reliability examinations were not performed due to the nature of the variable (observed child's behavior) that could not allow repeated examination. This may have brought in some observer bias although every effort was made to be consistent with the patient's ratings. It ought to anyway be noted that behavior modification utilized by the dentist could have influenced the behavior outcome, which was unavoidable.

A suggested recommendation for further research is to conduct more in-depth research on the behavior aspect in multiple visits in a larger population size.

\section{Conclusion}

This study demonstrates a possible relation between breakfast consumption and behavior changes in young children. With in the scope of the study and the variables, it can be recommended to consume breakfast before dental appointments unless otherwise specified, in order to eliminate many behavior-related problems during the course of treatment.

\section{References}

[1]. O'Neil CE, Byrd-Bredbenner C, Hayes D, Jana L, Klinger SE, StephensonMartin S. The role of breakfast in health: definition and criteria for a quality breakfast. J Acad Nutr Diet. 2014 Dec;114(12 Suppl):S8-S26. Pubmed PMID: 25458994.

[2]. Farshchi HR, Taylor MA, Macdonald IA. Beneficial metabolic effects of regular meal frequency on dietary thermogenesis, insulin sensitivity, and fasting lipid profiles in healthy obese women. Am J Clin Nutr. 2005 Jan;81(1):1624. Pubmed PMID: 15640455.

[3]. Chugani HT. A critical period of brain development: studies of cerebral glucose utilization with PET. Prev Med. 1998 Mar-Apr;27(2):184-8. Pubmed PMID: 9578992.

[4]. Benton D, Maconie A, Williams C. The influence of the glycaemic load of breakfast on the behaviour of children in school. Physiol Behav. 2007 Nov 23;92(4):717-24. Pubmed PMID: 17617427.

[5]. Thorleifsdottir B, Björnsson JK, Benediktsdottir B, Gislason T, Kristbjarnarson $\mathrm{H}$. Sleep and sleep habits from childhood to young adulthood over a 10-year period. J Psychosom Res. 2002 Jul;53(1):529-37. PubmedPMID: 12127168.

[6]. Cooper SB, Bandelow S, Nute ML, Morris JG, Nevill ME. Breakfast glycaemic index and exercise: combined effects on adolescents' cognition. Physiol Behav. 2015 Feb;139:104-11. Pubmed PMID: 25446221. 
[7]. Sandercock GR, Voss C, Dye L. Associations between habitual schoolday breakfast consumption, body mass index, physical activity and cardiorespiratory fitness in English schoolchildren. Eur J Clin Nutr. 2010 Oct;64(10):1086-92. Pubmed PMID: 20683459.

[8]. Hoyland A, Dye L, Lawton CL. A systematic review of the effect of breakfast on the cognitive performance of children and adolescents. Nutr Res Rev. 2009 Dec;22(2):220-43. Pubmed PMID: 19930787.

[9]. Deshmukh-Taskar PR, Radcliffe JD, Liu Y, Nicklas TA. Do breakfast skipping and breakfast type affect energy intake, nutrient intake, nutrient adequacy, and diet quality in young adults? NHANES 1999-2002. J Am Coll Nutr. 2010 Aug;29(4):407-18. PubmedPMID: 21041816.

[10]. Cooper SB, Bandelow S, Nevill ME. Breakfast consumption and cognitive function in adolescent schoolchildren. Physiol Behav. 2011 Jul 6;103(5):4319. PubmedPMID: 21439306

[11]. Widenhorn-Müller K, Hille K, Klenk J, Weiland U. Influence of having breakfast on cognitive performance and mood in 13- to 20-year-old high school students: results of a crossover trial. Pediatrics. 2008 Aug;122(2):27984. PubmedPMID: 18676544.

[12]. Adolphus K, Lawton CL, Dye L. The effects of breakfast on behavior and academic performance in children and adolescents. Front Hum Neurosci. 2013 Aug 8;7:425. Pubmed PMID: 23964220.

[13]. Witbracht M, Keim NL, Forester S, Widaman A, Laugero K. Female breakfast skippers display a disrupted cortisol rhythm and elevated blood pressure. Physiol Behav. 2015 Mar 1;140:215-21. PubmedPMID: 25545767.

[14]. Kulkarni VK, Kandya A, Arora S, Singh G. Decreased sleep in children and their behavioral problems in dental operatory. J Indian Soc Pedod Prev Dent. 2017 Apr-Jun;35(2):123-127. Pubmed PMID: 28492190.

[15]. Govindaraju L, Jeevanandan G, Subramanian EM. Knowledge and practice of rotary instrumentation in primary teeth among indian dentists: A questionnaire survey. Journal of International Oral Health. 2017 Mar 1;9(2):45.

[16]. Govindaraju L, Jeevanandan G, Subramanian E. Clinical Evaluation of Quality of Obturation and Instrumentation Time using Two Modified Rotary File Systems with Manual Instrumentation in Primary Teeth. J Clin Diagn Res. 2017 Sep;11(9):ZC55-ZC58. Pubmed PMID: 29207834.

[17]. Panchal V, Jeevanandan G, Subramanian E. Comparison of instrumentation time and obturation quality between hand K-file, H-files, and rotary Kedo-S in root canal treatment of primary teeth: A randomized controlled trial. J Indian Soc Pedod Prev Dent. 2019 Jan-Mar;37(1):75-79. Pubmed PMID: 30804311 .

[18]. Lakshmanan L, Mani G, Jeevanandan G, Ravindran V, Ganapathi SE. Assessing the quality of root canal filling and instrumentation time using kedo-s files, reciprocating files and k-files. Brazilian Dental Science. 2020 Jan 31;23(1):7-p.

[19]. Ravikumar D, Jeevanandan G, Subramanian EMG. Evaluation of knowledge among general dentists in treatment of traumatic injuries in primary teeth: A cross-sectional questionnaire study. Eur J Dent. 2017 AprJun;11(2):232-237. Pubmed PMID: 28729799.

[20]. Ramakrishnan M, Dhanalakshmi R, Subramanian EMG. Survival rate of different fixed posterior space maintainers used in Paediatric Dentistry - A systematic review. Saudi Dent J. 2019 Apr;31(2):165-172. Pubmed PMID: 30983825 .

[21]. Subramanyam D, Gurunathan D, Gaayathri R, Vishnu Priya V. Comparative evaluation of salivary malondialdehyde levels as a marker of lipid peroxidation in early childhood caries. Eur J Dent. 2018 Jan-Mar;12(1):67-70. Pubmed PMID: 29657527.
[22]. Vishnu Prasad S, Kumar M, Ramakrishnan M, Ravikumar D. Report on oral health status and treatment needs of 5-15 years old children with sensory deficits in Chennai, India. Spec Care Dentist. 2018 Jan;38(1):58-59. Pubmed PMID: 29333605.

[23]. Jeevanandan G, Ganesh S; Arthilakshmi. Kedo file system for root canal preparation in primary teeth. Indian J Dent Res. 2019 Jul-Aug;30(4):622-624. Pubmed PMID: 31745062.

[24]. Ramadurai N, Gurunathan D, Samuel AV, Subramanian E, Rodrigues SJL. Effectiveness of $2 \%$ Articaine as an anesthetic agent in children: randomized controlled trial. Clin Oral Investig. 2019 Sep;23(9):3543-3550. Pubmed PMID: 30552590.

[25]. Panchal V, Gurunathan D, Shanmugaavel AK. Smartphone application as an aid in determination of caries risk and prevention: A pilot study. Eur J Dent. 2017 Oct-Dec;11(4):469-474. Pubmed PMID: 29279672.

[26]. Vignesh R, Sharmin D, Rekha CV, Annamalai S, Baghkomeh PN. Management of Complicated Crown-Root Fracture by Extra-Oral Fragment Reattachment and Intentional Reimplantation with 2 Years Review. Contemp Clin Dent. 2019 Apr-Jun;10(2):397-401. Pubmed PMID: 32308309.

[27]. Shao AG, Kahabuka FK, Mbawalla HS. Children's Behaviour in the Dental Setting according to Frankl Behaviour Rating and their Influencing Factors. J Dental Sci. 2016;1(1):000103.

[28]. Mishra G, Thakur S, Singhal P, Ghosh SN, Chauhan D, Jayam C. Assessment of child behavior in dental operatory in relation to sociodemographic factors, general anxiety, body mass index and role of multi media distraction. J Indian Soc Pedod Prev Dent. 2016 Apr-Jun;34(2):159-64. Pubmed PMID: 27080967.

[29]. Kleinman RE, Murphy JM, Little M, Pagano M, Wehler CA, Regal K, Jellinek MS. Hunger in children in the United States: potential behavioral and emotional correlates. Pediatrics. 1998 Jan;101(1):E3. Pubmed PMID: 9417167.

[30]. Lakhani B, Indushekar KR, Shalini G, Nayanika S, Ekta T. Behavior Assessment Using Frankl Rating Scale and Identification of Personality in Pediatric Dental Operatory. J Child Adolesc Behav 5(5): 356.

[31]. Jamali Z, Najafpour E, Ebrahim Adhami Z, Sighari Deljavan A, Aminabadi NA, Shirazi S. Does the length of dental procedure influence children's behavior during and after treatment? A systematic review and critical appraisal. J Dent Res Dent Clin Dent Prospects. 2018 Winter;12(1):68-76. Pubmed PMID: 29732024.

[32]. Aminabadi NA, Ghoreishizadeh A, Ghoreishizadeh M, Oskouei SG, Ghojazadeh M. Can child temperament be related to early childhood caries? Caries Res. 2014;48(1):3-12. Pubmed PMID: 24216506.

[33]. Sharath A, Rekka P, Muthu MS, Rathna Prabhu V, Sivakumar N. Children's behavior pattern and behavior management techniques used in a structured postgraduate dental program. J Indian Soc Pedod Prev Dent. 2009 JanMar;27(1):22-6. Pubmed PMID: 19414970.

[34]. Wilson S. Sedation for the Pediatric Patient. Behavior Management in Dentistry for Children 2014; 131-143.

[35]. Fugas V, Berta E, Walz F, Fortino A, Martinelli MJ. Breakfast habit and quality in students from two public primary schools in the city of Santa Fe. Arch Argent Pediatr. 2013 Dec 1;111(6):502-7.

[36]. Shinohara S, Nomura Y, Shingyouchi K, Takase A, Ide M, Moriyasu K, et al. Structural relationship of child behavior and its evaluation during dental treatment. J Oral Sci. 2005 Jun;47(2):91-6. Pubmed PMID: 16050489. 